\title{
Synthesis, characterizations and hydrophobicity of micro/nano scaled heptadecafluorononanoic acid decorated copper nanoparticle
}

\author{
Hung-Hsia Chen ${ }^{1}$, R. Anbarasan ${ }^{1}$, Long-Sheng Kuo1, Meng-Yu Tsai', Ping-Hei Chen ${ }^{1,}{ }^{*}$ and Kuei-Feng \\ Chiang ${ }^{2}$
}

Copper nanoparticle was synthesized in the presence of heptadecafluorononanoic acid by the conventional solution immersion method at room temperature from the copper plate, as a resource material. The bulk etching rate was calculated by the weight loss method. The pale green colored Cu-HDFN was characterized by Fourier transform infrared spectroscopy, UV-Visible spectroscopy, $\mathrm{X}$-ray photoelectron spectroscopy, scanning electron microscopy, transmission electron microscopy and contact angle measurements and the results are critically analyzed.

Keywords: Copper nanoparticle; Etching; Hydrophobicity

Citation: Hung-Hsia Chen, R. Anbarasan, Long-Sheng Kuo, Meng-Yu Tsai, Ping-Hei Chen and Kuei-Feng Chiang, "Synthesis, characterizations and hydrophobicity of micro/nano scaled heptadecafluorononanoic acid decorated copper nano particle", Nano-Micro Lett. 2, 101-105 (2010). doi: 10.5101/nml.v2i2.p101-105

Synthesis of nanosized material from the bulk resource material is a fascinating field of research because of its novelty and application in various science and engineering fields. Various techniques are employed for the generation of nanosized material from its bulk one [1-10]. Generally, thermal treatments are employed for this purpose, unfortunately it requires costly instruments. In order to outwit this problem our research team has followed a conventional chemical method. The advantages of the present investigations are 1)economically cheaper, 2)no use of expensive and hazardous chemicals and hence it is a pollution free method, 3)the resultant material has hydrophobic characters and 4) the simultaneous coating of hydrophobic material on the surface of the bulk resource material. A modified copper surface with super hydrophobic characteristics could be used either in vapor chamber or heat pipe that has been widely employed for heat spreading of electric devices.
Recently, Song et al [11] reported about the electroless replacement deposition method for the generation of super hydrophobic copper substrate. Super hydrobhopic surfaces based on copper hydroxide nano needles were reported by $\mathrm{Wu}$ and co-workers [12]. Wang and his research team [13] studied the super hydrophobic copper films. The super hydrophobic character was supported by water contact angle measurement reports. Super hydrophobic $\mathrm{Cu}(\mathrm{OH})_{2}$ nano tubes from copper foil were reported in the literature [14]. Other authors also reported about the super hdyrophobic copper plate surface by different techniques [15-17]. Fluoro compounds exhibit hydrophobic character with water surface and this urged us to do the surface modification of copper plate by heptadecafluorononanoic acid (HDFNA). By thorough literature survey, we could not find any report based on the synthesis of $\mathrm{Cu}$ nano particle in the presence of HDFNA. In the present investigation we took this job as a challenge and

1 Department of Mechanical Engineering, National Taiwan University, Taipei-10617, Taiwan

2 Thermal division, Advanced Vital Component Company, Taiwan

${ }^{*}$ Corresponding author. Email: phchen@ntu.edu.tw 
successfully synthesized the $\mathrm{Cu}$ nano particle in the presence of HDFNA with simultaneous coating on the same in ethanol medium at room temperature for the first time, with Fourier transform infrared (FTIR) spectroscopy, UV-Visible spectroscopy (UV-vis), X-ray photoelectron spectroscopy (XPS), scanning electron microscopy (SEM), transmission electron microscopy (TEM) and contact angle measurement reports. When compared with the literature reports, the present investigation yielded an economically cheaper method to produce the nanosized copper from the resource material with hydrophobic character. Moreover, this investigation didn't use any hazardous or toxic solvents to produce nanosized copper and hence is an eco-friendly method.

\section{Experiment}

\section{Materials}

HDFNA (Fluka, USA) and ethanol (Sigma Aldrich, USA) were purchased and used as received. A copper plate with the purity of $99.99 \%$ was used as a resource material for the generation of copper nano particle. The dimensions of the copper plate are mentioned below: weight of copper plate-4.1310 g, length- $6 \mathrm{~cm}$, breadth- $2 \mathrm{~cm}$, area- $12 \mathrm{~cm}^{2}$.

\section{Surface treatment and etching rate of the copper plate}

The copper plate with the above mentioned dimensions was rubbed with metal paper and ultrasonicated for $1 \mathrm{~h}$ to remove the metal oxide from the surface. $50 \mathrm{~mL}$ of double distilled water (DD) was used to wash the surface of the copper plate. $0.10 \mathrm{~g}(0.02 \mathrm{M})$ of HDFNA was dissolved in $100 \mathrm{~mL}$ of ethanol in a $150 \mathrm{~mL}$ beaker under mild stirring condition in the open air atmosphere. Accurately weighed and dried copper plate was immersed in the reaction medium with mild stirring under the influence of atmospheric air, which activated the chemical etching process. After 12 hours of etching, the copper plate was removed from the reaction medium and dried at $80^{\circ} \mathrm{C}$ for $1 \mathrm{~h}$ in an air oven. The dried copper plate was weighed accurately and change in mass was tabulated. Meanwhile, 2 $\mathrm{mL}$ of the aliquot was taken from the reaction medium and the UV-Visible absorbance was measured. Both the weight and absorbance measurements were carried out in a regular period of time to measure the etching rate. The bulk etching rate $\left(V_{B}\right)$ was calculated from the following equation.

$$
V_{B}=\frac{\Delta_{m}}{2 A \rho t}
$$

Where $\Delta_{m}$ is the mass difference, $A$ is the etched surface area, $\rho$ is the density of the copper plate and $t$ is the etching time. At the end of the etching reaction, the etched copper plate was removed from the beaker and the content of the beaker was diluted with water and frozen below $0^{\circ} \mathrm{C}$ in the refrigerator. Thus frozen contents were dried under vacuum by using a freeze drier. After 48 hours of freeze drying, the pale green mass obtained was HDFNA coated copper nano particle and the product was subjected to analytical characterizations. 3 $\mathrm{mg}$ of the $\mathrm{Cu}-\mathrm{HDFN}$ sample was made disk with $200 \mathrm{mg}$ of spectral grade $\mathrm{KBr}$ and the FTIR spectrum was measured between 400 and $4000 \mathrm{~cm}^{-1}$ with the help of Perkin Elmer Spectrum 100 series instrument. Jasco V-570 instrument was used for UV-Visible spectrum measurement. Surface morphology of the sample was measured by JSM 6300, Jeol product, SEM instrument. The size of the copper nano particle was determined by TEM 3010, a product of JEOL. The binding energy of HDFNA decorated copper nano particle was determined by XPS (XPS, Thermo Scientific, Theta Probe, UK). The contact angle of $\mathrm{Cu}-\mathrm{HDFN}$ coated copper plate was determined by VCA 2500, Taiwan instrument.

\section{Experimental results and discussion FTIR study}

Figure 1a shows the FTIR spectrum of $\mathrm{Cu}-\mathrm{HDFN}$. A peak at $546 \mathrm{~cm}^{-1}$ confirmed the presence of $\mathrm{F}$ in $\mathrm{Cu}-\mathrm{HDFN}$. A twin peak at $656 \mathrm{~cm}^{-1}$ indicated the $\mathrm{Cu}-\mathrm{O}$ stretching vibration. The metal salt $(\mathrm{Cu}-\mathrm{O}-\mathrm{C})$ peak is appeared at $1144 \mathrm{~cm}^{-1}$. The metal salt carbonyl group is appeared at $1689 \mathrm{~cm}^{-1}$. Generally the ester carbonyl group will appear at $1725 \mathrm{~cm}^{-1}$. In the present investigation, the ester carbonyl peak was red shifted due to the smaller size and highly electron withdrawing nature of $\mathrm{F}$ atom

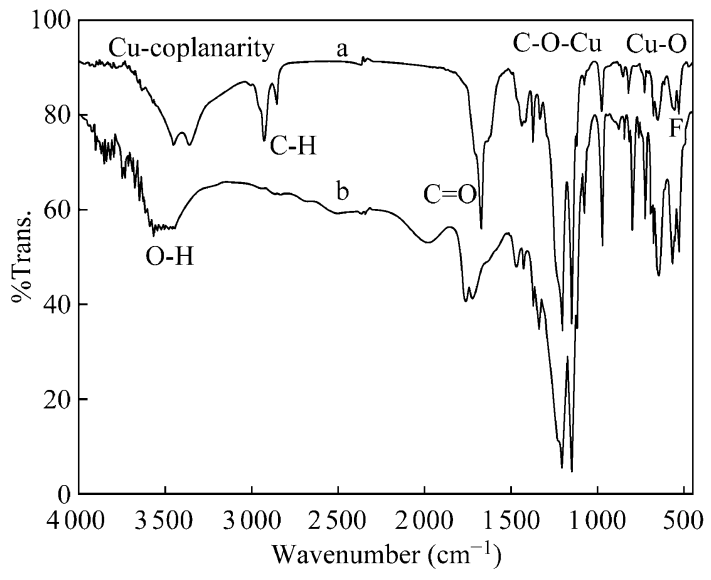

FIG. 1. FTIR spectrum of HDFNA decorated copper nano particle. 
in the HDFNA backbone. The $\mathrm{C}-\mathrm{H}$ symmetric and anti-symmetric stretching is observed at 2856 and $2932 \mathrm{~cm}^{-1}$ respectively. A twin peak around $3400 \mathrm{~cm}^{-1}$ declared the presence of $\mathrm{Cu}$ co-planarity. An ionic interaction is existing between the copper nanoparticle and HDFNA. Thus the FTIR spectrum confirmed the various functionalities presented in the HDFNA coated copper nano particle. For the sake of comparison, the FTIR spectrum of pure HDFNA is shown in Fig. 1 as 1 b. The important peaks are characterized below: A peak at $3752 \mathrm{~cm}^{-1}$ indicates the presence of intermolecular hydrogen bonding due to the carboxyl group of HDFNA. A broad peak around $3500 \mathrm{~cm}^{-1}$ is responsible for the carboxyl $\mathrm{OH}$ stretching. Generally, the long chain carboxylic acid will not exhibit the C-H stretching around $2900 \mathrm{~cm}^{-1}$ in its acidic form. The carbonyl peak is observed as a doublet around 1725 $\mathrm{cm}^{-1}$ due to the free and hydrogen bonded carboxyl group. By comparing Fig. 1a and b, appearance of $\mathrm{Cu}$ co-planarity, $\mathrm{C}-\mathrm{H}$ stretching, sharp $\mathrm{C}=\mathrm{O}$ stretching, $\mathrm{C}-\mathrm{O}-\mathrm{Cu}$ and $\mathrm{Cu}-\mathrm{O}$ stretching confirmed the formation of $\mathrm{Cu}-\mathrm{HDFN}$.

\section{UV-Visible spectroscopy}

Figure 2 indicates the UV-Visible spectrum of $\mathrm{Cu}-\mathrm{HDFN}$. A negative absorbance peak was observed at $322 \mathrm{~nm}$. This peak is quite natural and due to the $\mathrm{n}$ to $\mathrm{Pi}^{*}$ transition [18] of the HDFNA decorated copper nano particle [19]. The hindered transition can be explained on the basis of highly negatively charged HDFNA decorated co-planarized copper nano particle. It is important to note that the absorbance value increases with the increase of etching time. After 24 hours of etching, the absorbance value drastically increased and thereafter it linearly increased. This explained the molecular oxygen influenced chemical etching process. After 24 hours of etching, the diffused copper reacted with the HDFNA in the presence of atmospheric air to produce the $\mathrm{Cu}-\mathrm{HDFN}$ and the same was coated on the copper plate. As a result of $\mathrm{Cu}$-HDFN coating on

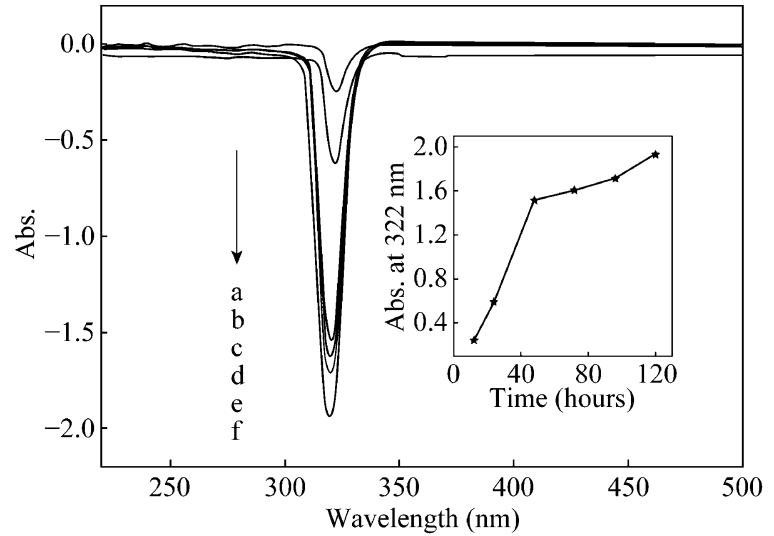

FIG. 2. UV-Visible spectrum of HDFNA decorated copper nano particle. the copper plate, the free surface available for the chemical etching is restricted and a slow increase in the chemical etching process is observed. The inset of Fig. 2 confirms the same concept. The plot was made between etching time and absorbance at $322 \mathrm{~nm}$. The plot showed a steep increase in absorbance up to 48 hours and then showed a linear increase with the etching time. This confirmed that up to 48 hours, the chemical etching was the only reaction occurred inside the reaction medium, after 48 hours, etching and coating were the competitive reactions. Hence, after 48 hours the absorbance value was linearly increased with the etching time.

\section{SEM report}

Figure 3 indicates the surface morphology of $\mathrm{Cu}-\mathrm{HDFN}$. Figure $3 \mathrm{a}$ represents the plate like morphology. Figure $3 \mathrm{~b}$ indicates the cauli flower like morphology. Flower like morphology is responsible for superhydrophobicity nature of a material [4]. The SEM results proved that the HDFNA decorated copper plate was slowly converted into super hydrophobic one due to the simultaneous coating of $\mathrm{Cu}-\mathrm{HDFN}$ on the copper plate. Further, this can be confirmed with the contact angle measurement and will be discussed in the forth coming sessions.
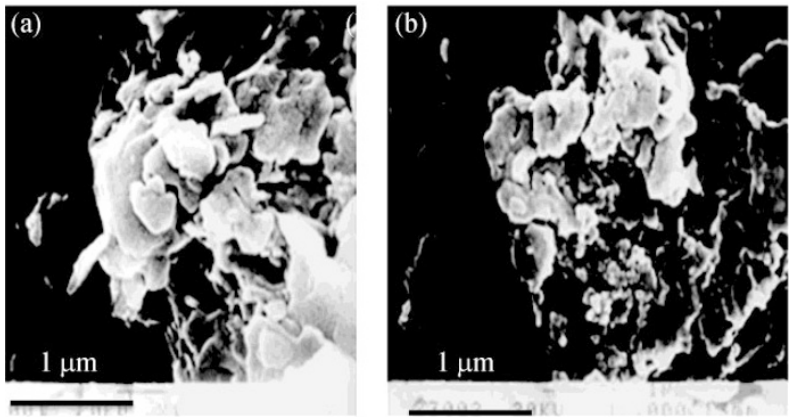

FIG. 3. SEM of HDFNA decorated copper nano particle

\section{TEM analysis}

Figure 4 shows the topography of HDFNA coated copper nano particle. Figure $4 \mathrm{a}$ represents the agglomerated form of copper nano particle with the size of $400 \mathrm{~nm}$. Figure $4 \mathrm{~b}$ represents the sphere topography of copper nano particle with the size of $10 \sim 65 \mathrm{~nm}$. Figure 4c indicates the individual nano particle size of $20 \mathrm{~nm}$ with the distorted sphere morphology. The distortion is due to the adsorption of HDFNA on the surface of the copper nano particle. The TEM images concluded that during the synthesis of super hydrophobic $\mathrm{Cu}$ plate, nanosized copper was diffused into the reaction medium from the copper plate resource material and formed HDFNA coated copper nano particle and the same was coated on the 


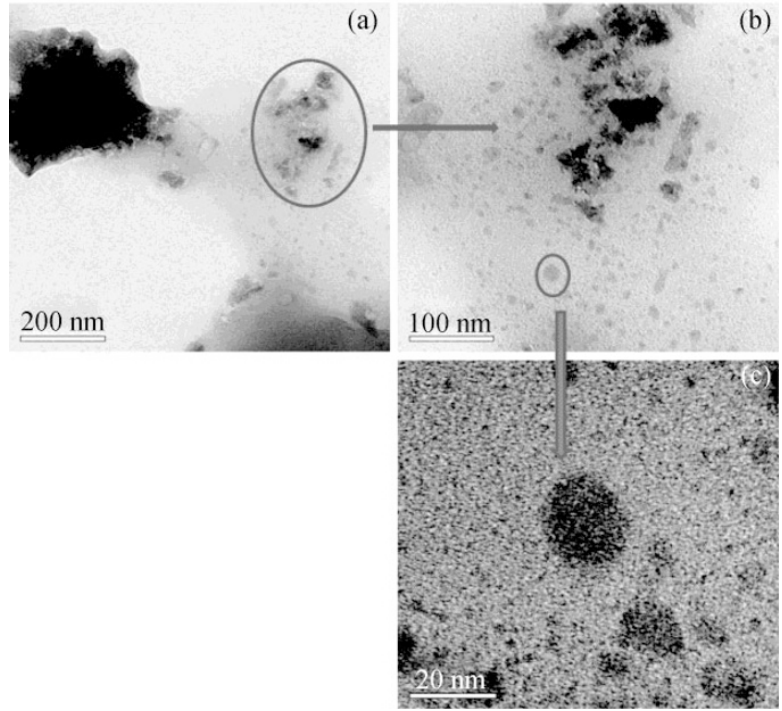

FIG. 4. TEM of HDFNA decorated copper nano particle.

surface of the resource material. This is an eco-friendly and economically cheaper method to produce the copper nano particle from the resource.

\section{XPS analysis}

Figure 5 shows the XPS of HDFNA decorated copper nano particle. It showed the binding energy of $\mathrm{Cu} 2 \mathrm{p}_{3 / 2}$ and $\mathrm{Cu} 2 \mathrm{p}_{1 / 2}$ at 933.08 and $950.2 \mathrm{eV}$ [20] respectively. A signal at $933.08 \mathrm{eV}$ confirmed the existence of $\mathrm{Cu}$ nano particle (zero oxidation state). Moreover, the \% of copper was found to be $1.07 \%$. The F1s level of $\mathrm{F}$ in HDFNA decorated copper nano particle was determined at $687.23 \mathrm{eV}$ with $35.92 \%$ content. The C1s and O1s peaks of HDFNA are observed at 281.9 and $530.8 \mathrm{eV}$ respectively. The XPS analysis concluded that the HDFNA is coated on the surface of the copper nano particle. Again this supported the core-shell like structure of HDFNA decorated copper nano particle.

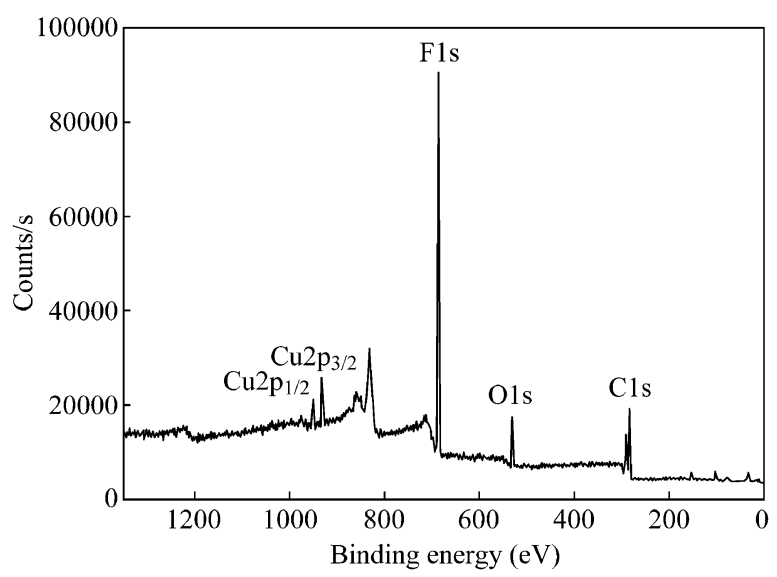

FIG. 5. XPS of HDFNA coated copper nano particle.

\section{Determination of bulk etching rate}

The bulk etching rate was determined from equation (1). It was interesting to note that while increasing the etching time, the colorless reaction medium turns into pale green. This ultimately indicated that the concentration of the diffused copper nano particle was increased. The etching rate was determined from the plot drawn between the etching time and the difference in mass (see Fig. 6a). The slope value was derived by linear fit method as $0.31 \mathrm{mg} / \mathrm{cm}^{2} . \mathrm{H}$. An important point noted here is that the weight loss after 24 hour is drastically increased. At the same time the concentration of copper in the reaction medium was increased and this can be further supported by the UV-Visible absorption value (i.e.) the absorption value increased with the increase of the etching time. In 1995, Bryce et al. reported about the bulk etching rate of copper in ferric chloride solution as $0.27 \mathrm{mg} / \mathrm{cm}^{2}$.s [5]. Our report is in accordance with them. Figure $6 \mathrm{~b}$ indicates the effect of etching time on the water contact angle, and discussed in the next session.

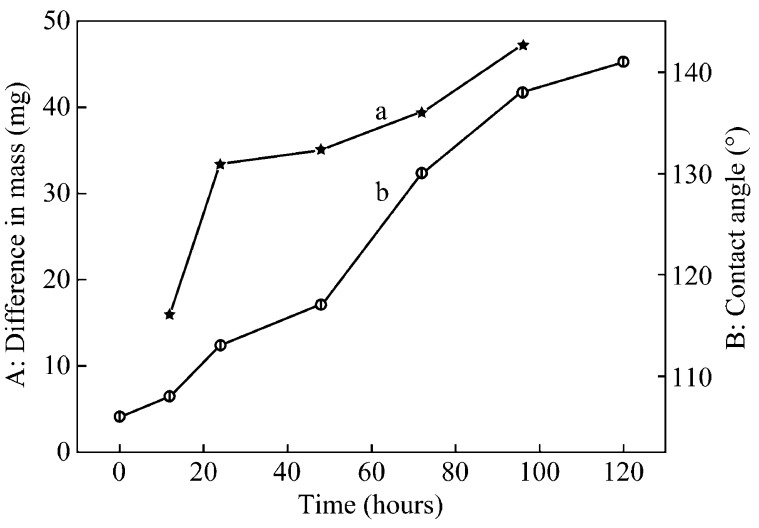

FIG. 6. Bulk etching rate of copper plate at different time interval.

\section{Contact angle measurement}

The contact angle measurement confirmed the hydrophobicity nature of the HDFNA coated copper nano particle. After 5 days of etching, the CA was determined as $140.7^{\circ}$. This proved the hydrophobic nature of the $\mathrm{Cu}-\mathrm{HDFN}$ coated copper plate at the $5^{\text {th }}$ day. Figure 7 shows the picture of $\mathrm{CA}$ measured for the $\mathrm{Cu}-\mathrm{HDFN}$ coated copper plate. Thus the contact angle co-supported the SEM morphology of HDFNA coated copper nano particle. While increasing the etching time, the water contact angle of copper plate was increased due to the chemical etching reaction. Figure $6 \mathrm{~b}$ represents the effect of etching time on the water contact angle. Due to the surface etching reaction, the water contact angle was increased. 


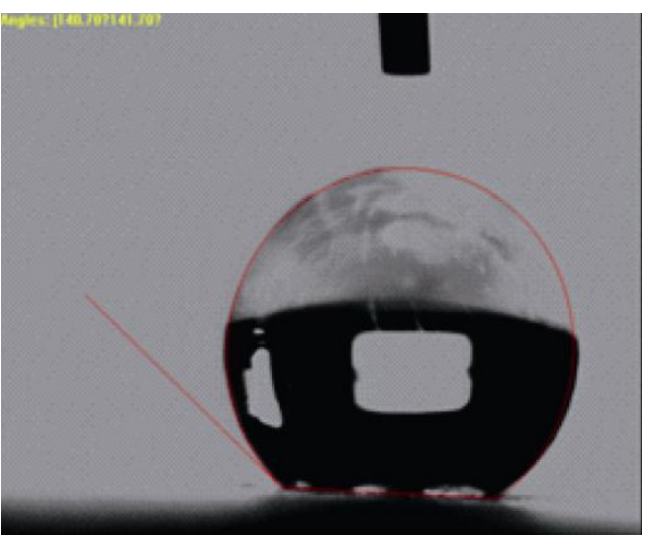

FIG. 7. Contact angle of $\mathrm{Cu}-\mathrm{HDFN}$ coated copper plate.

\section{Conclusions}

The important points are presented here as conclusions. 1) The HDFNA decorated copper nano particle was successfully synthesized with the C.A of $140.7^{\circ}$. The formation of $\mathrm{Cu}-\mathrm{HDFN}$ was confirmed by FTIR spectroscopy by noting a peak at 656 $\mathrm{cm}^{-1}$. 2) Further, the presence of copper in Cu-HDFN was confirmed by UV-Vis spectroscopy at an absorbance value of $322 \mathrm{~nm} .3$ ) The absorbance value increased with the increase of etching time. 4) The bulk etching rate was determined as 0.31 $\mathrm{mg} / \mathrm{cm}^{2}$. H. 5) XPS analysis confirmed the binding energy of $\mathrm{Cu} 2 \mathrm{p}_{3 / 2}$ at $933.08 \mathrm{eV}$. SEM (cauli flower morphology), C.A measurement $\left(140.7^{\circ}\right)$ and TEM (10 65 nm size) combinedly declared that the HDFNA coated copper nano particle is a suitable candidate for the manufacture of copper nano particle with hydrophobic character. The above points inferred that our methodology yielded an economically cheaper and eco-friendly method with the nanosized copper particle.

Received 29 March 2010; accepted 5 May 2010; published online 20 May 2010.

\section{References}

1. W. Z. Jia, E. Reitz, H. Sun, H. Zhang and Y. Lei, Mater. Lett. 63, 519 (2009). doi:10.1016/j.matlet.2008.10.053.

2. J. M. Xi, L. Feng and L. Jiang, App. Phys. Lett. 92, 1 (2008). doi:10.1063/1.2839403.

3. X. Li and M. X. Wan, Cryst. Grow. Des. 6, 2661 (2006). doi: $10.1012 / \operatorname{cg} 0506440$.

4. Z. W. Cao, D. B. Xiao, L. T. Kang, Z. L. Wang, S. X. Zhang, Y. Ma, H. B. Hu and J. N. Yao, Chem. Commun. 23, 2692 (2008). doi:10.1039/b803959c.
5. C. Bryce and D. Berk, Ind. Eng. Chem. Res. 34, 1412 (1995).

6. C. T. Hsieh, J. M. Chen, H. H. Lin and H. C. Shih, Appl. Phys. Lett. 82, 3316 (2003). doi:10.1063/1.15690943.

7. Y. W. Zhu, T. Yu, F. C. Cheong, X. J. Xui, C. T. Lim, V. B. C. Tan, J. T. L. Thong and C. H. Sow, Nanotechnology 16, 88 (2005). doi:10.1088/0957-44841 16/1/018.

8. W. H. Wang, Y. J. Zhan and G. H. Wang, Chem. Commun. 8, 727 (2001). doi: 10.1039/b008215p.

9. C. K. Xu, Y. K. Liu, G. D. Xu and G. H. Wang, Mater. Res. Bull. 37, 2365 (2002).

10. Z. H. Yang, J. Z. Xu, W. X. Zhang, A. P. Liu and S. P. Tang, J. Solid State Chem. 180, 1390 (2007). doi:10.1016/j.jssc.2007.02.008.

11. W. Song, J. J. Zhang, Y. F. Xie, Q. Cong and B. Zhao, J. Colloid Interface Sci. 329, 208 (2009). doi:10.1016/j.jcis. $\underline{2008.09 .059}$

12. X. F. Wu and G. Q. Shi, J. Phys. Chem. B 110, 11247 (2006). doi:10.1012/jp 056969x

13. S. T. Wang, L. Feng, H. Liu, T. L. Sun, X. Zhang, L, Jiang and D. B. Zhu, Chem. Phys. Chem. 6, 1475 (2005). doi:10.1002/cphc.200500204.

14. X. H. Chen, L. H. Kong, D. Dong, G. B. Yang, L. G. Yu, J. M. Chen and P. Y. Zhang, Appl. Surf. Sci. 255, 4015, (2009). doi: 10.1016/j.appsursci.4015-4019.

15. S. T. Wang, L. Feng and L. Jiang, Adv. Mater. 18, 767 (2006). doi:10.1002/adma.2005.01794.

16. X. C. Jiang, T. Herricks and Y. N. Xia, Nano Lett. 2, 1333 (2002). doi:10.1021/n10257519.

17. L.S. Huang, S. G. Yang, T. Li, B. X. Gu, Y. W. Du, Y. N. $\mathrm{Lu}$ and S. Z. Shi, J. Cryst. Growth. 260, 130 (2004). doi:10.16/j.j.crystgro.2003.08.012.

18. Y. Kaya, H. Mutlu and G. Irex, G. U. J. Sci. 23, 13 (2010).

19. P. K. Khanna, T. S. Kale, M. Shaikh, N.K. Rao and C. V. V. Satyanarayana, Mat. Chem. Phys. 110, 21 (2008). doi:10.1016/j.mat.chem.phys.2008.01.013.

20. N. Cioffi, L. Torsi, N. Ditaranto, G. Tantillo, L. Ghibelli, L. Sabbatini, T. Bleve-Zacheo, M. D'Alessio, P. G. Zambonin and E. Traversa, Chem. Mater. 2005, 17, 5255 (2005). doi:10.1021/cm0505244. 